\title{
Accounting for uncertainty from zero inflation and overdispersion in paleoecological studies of predation using a hierarchical Bayesian framework
}

\author{
Jansen A. Smith*广 (D), John C. Handley ${ }^{\dagger}$, and Gregory P. Dietl
}

\begin{abstract}
The effects of overdispersion and zero inflation (e.g., poor model fits) can result in misinterpretation in studies using count data. These effects have not been evaluated in paleoecological studies of predation and are further complicated by preservational bias and time averaging. We develop a hierarchical Bayesian framework to account for uncertainty from overdispersion and zero inflation in estimates of specimen and predation trace counts. We demonstrate its application using published data on drilling predators and their prey in time-averaged death assemblages from the Great Barrier Reef, Australia.

Our results indicate that estimates of predation frequencies are underestimated when zero inflation is not considered, and this effect is likely compounded by removal of individuals and predation traces via preservational bias. Time averaging likely reduces zero inflation via accumulation of rare taxa and events; however, it increases the uncertainty in comparisons between assemblages by introducing variability in sampling effort. That is, there is an analytical cost with time-averaged count data, manifesting as broader confidence regions. Ecological inferences in paleoecology can be strengthened by accounting for the uncertainty inherent to paleoecological count data and the sampling processes by which they are generated.

Jansen A. Smith. GeoZentrum Nordbayern, Friedrich-Alexander University Erlangen-Nürnberg (FAU), Erlangen, Bayern 91054, Germany; Paleontological Research Institution, Ithaca, New York 14850, U.S.A. E-mail: jansen. smith@fau.de

John C. Handley. Goergen Institute for Data Science, University of Rochester, Rochester, New York 14627, U.S.A.; Paleontological Research Institution, Ithaca, New York 14850, U.S.A. E-mail: john.handley@rochester.edu

Gregory P. Dietl. Paleontological Research Institution, Ithaca, New York 14850, U.S.A.; Department of Earth and Atmospheric Sciences, Cornell University, Ithaca, New York 14853, U.S.A. E-mail: gpd3@cornell.edu
\end{abstract}

Accepted: 23 July 2021

*Corresponding author.

$\dagger$ These authors contributed equally to this work.

\section{Introduction}

In the last three decades, accounting for overdispersion and zero inflation in ecological studies that use count data has become increasingly common (e.g., Welsh et al. 1996; Martin et al. 2005; Warton 2005; Sileshi 2008; Wenger and Freeman 2008; Millar 2009; Sólymos et al. 2012; Dénes et al. 2015; Blasco-Moreno et al. 2019). With count data, overdispersion occurs when the variance of the data is significantly larger than the mean, indicating the model being applied does not appropriately capture the variance in the data (Bliss and Fisher 1953). Zero inflation, the presence of excess zeros in a dataset compared with the number of zeros expected under commonly applied count distributions (e.g., binomial, Poisson; Lambert 1992; Heilbron 1994), is one process that can produce overdispersion. These excess zeros, which can be ecologically real (i.e., true) or produced by non-ecological processes (i.e., false; see Table 1 for paleoecological examples), can change the model that provides the best fit to data and can lead to erroneous conclusions drawn from poorly fit models (MacKenzie et al. 2002; Martin et al. 2005; BlascoMoreno et al. 2019).

True zeros have ecological explanations, such as a species not occurring at a particular site for 
TABLE 1. Potential types of zeros in paleoecological count data with respect to those identified in ecological studies. When taking a sample to evaluate the ecology of a community, ecological processes (i.e., true zeros) and sampling artifacts (i.e., false zeros) can distort the resulting sample relative to the true distribution of specimens or predation events. In paleoecological samples, distortions from additional factors (e.g., preservational bias, time averaging) must also be addressed. *Following Blasco-Moreno et al. (2019). ${ }^{\dagger}$ The possibility of a zero count for a predation trace is predicated on the presence of the prey taxa in the assemblage $\left(n_{i}>1\right)$. When testing a hypothesis on multiple prey taxa, we must also assume the sample from the assemblage is representative of the set of prey taxa that could possibly have been sampled (i.e., were present in the community over the duration of time averaging; see Table 2).

\begin{tabular}{lcc}
\hline \hline $\begin{array}{l}\text { Count data } \\
\text { source }\end{array}$ & $\begin{array}{c}\text { Zero classifications } \\
\text { used for ecological } \\
\text { data* }^{*}\end{array}$ & $\begin{array}{c}\text { Zero classifications used } \\
\text { here for paleoecological } \\
\text { data }\end{array}$ \\
\hline
\end{tabular}

Specimens True Ecological

Description for zeros in paleoecological data

False Sampling

- Preservational

Predation traces $^{\dagger}$
Ecological

cological

Preservational

$\begin{array}{cc}\text { False } & \text { Error } \\ \text { Random } & \text { Sampling }\end{array}$

Prey type did not occupy the habitat(s) represented in the assemblage, for the duration over which the assemblage was time-averaged, due to ecological processes or as a result of the effect being studied (e.g., environmental tolerance).

Prey type did occupy the habitat(s) represented in the assemblage but was not present at a particular location by chance (e.g., patchiness), for the duration over which the assemblage was time-averaged.

Insufficient sampling effort resulted in failure to collect a prey type that was present in the assemblage (e.g., rarity).

Prey type removed from the assemblage by postmortem taphonomic processes (e.g., fragmentation, dissolution, winnowing).

Prey type was not preyed upon because it and the predator occupied different habitats.

Prey type occurred in the same habitat as the predator but was not preyed upon (e.g., more highly preferred prey was available, the prey type was avoided).

Prey type was sufficiently rare in the habitat and so was not encountered by the predator.

Predation trace(s) removed from the assemblage by postmortem taphonomic processes (e.g., reworking before burial, compaction during lithification).

Predation trace(s) attributed to wrong predator and excluded from analysis.

Zero is due to the small sample size of a prey type relative to the predation frequency on that prey type (i.e., low on preference hierarchy and uncommon in the community). an ecological reason (e.g., competitive exclusion) or because, by chance, the species does not saturate its entire suitable range (Martin et al. 2005) the latter is similar to a true, "random zero," described by Blasco-Moreno et al. (2019). False zeros occur when the investigator errs. Errors might include design elements of the study (e.g., attempting to sample a species that is not present in a particular habitat) or failure to observe a species when it is present, which may be common for rare or cryptic species (Welsh et al. 1996; Martin et al. 2005; Wenger and Freeman 2008; Blasco-Moreno et al. 2019). Accounting for these zeros and differentiating between zeros from different sources can change the results of a study.

Blasco-Moreno et al. (2019) demonstrated this possibility in their examination of the effects of overdispersion and zero inflation on ecological inference. Rather than count data for individuals of different species in a habitat, Blasco-Moreno et al. (2019) used counts of herbivory traces on the flowering heads of four plant species in the genus Senecio, including two native and two exotic species, to evaluate the enemy release hypothesis - that introduced or exotic species will experience less predation compared with native species because they "left behind" their 
TABLE 2. Common assumptions for time-averaged count data in paleoecological studies of molluscan predator-prey interactions.

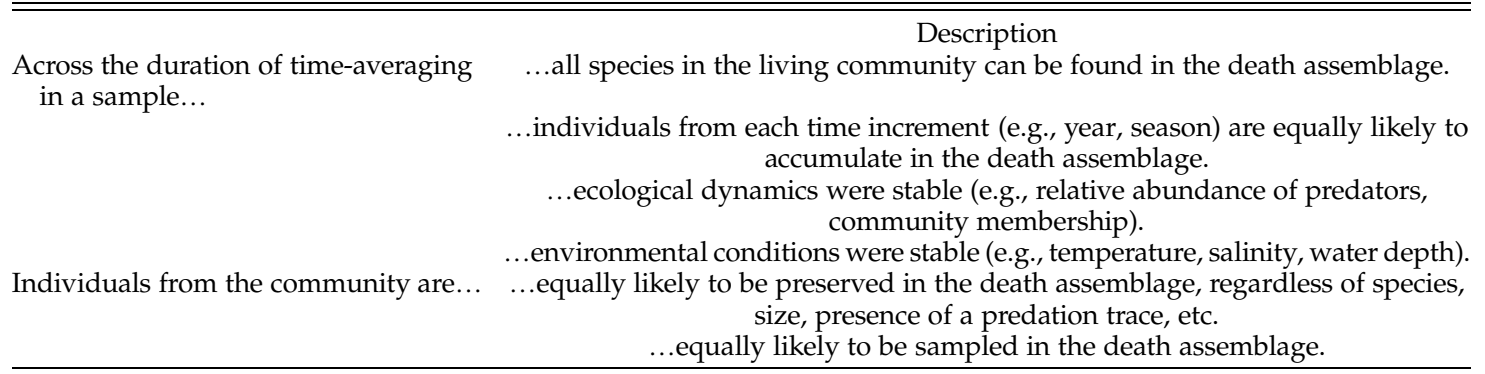

enemies (Keane and Crawley 2002). Depending on the model used, the Senecio herbivory data could be taken to support or reject the enemy release hypothesis. Using Poisson and zero-inflated Poisson models, Blasco-Moreno et al. (2019) found support for the enemy release hypothesis; however, when utilizing negative binomial and zero-inflated negative binomial models, the hypothesis was rejected. As the zero-inflated negative binomial model was the best-fitting model-it accounted for overdispersion better than the zero-inflated Poisson model, likely because negative binomial models relax the requirement of an equal variance and mean - the authors concluded that the enemy release hypothesis should be rejected. Choosing the correct model to account for overdispersion and zero inflation is critical for reaching correct conclusions from ecological count data.

Just as in ecological studies, count data used in paleoecological studies are susceptible to overdispersion and zero inflation. Though some investigators have begun to consider overdispersion (e.g., Martinelli et al. 2015), the potentially misleading effects of zero inflation have not been explicitly addressed. Here we present a hierarchical Bayesian framework consistent with ecological studies as a first step toward addressing overdispersion and zero inflation in paleoecological studies, with consideration for the unique features of paleoecological count data (e.g., time averaging). We apply this framework to a dataset that combines two previously published paleoecological datasets from the Great Barrier Reef - one on molluscan predator-prey interactions (Martinelli et al. 2015) and another on the degree of time averaging in an assemblage (Kosnik et al. 2009; see "A Paleoecological Case Study")- to demonstrate the potential effects of overdispersion and zero inflation on the results drawn from paleoecological count data.

In addition to applying zero-inflated models in a new context, under a new set of assumptions and conditions, we extend the modeling approach to include zero inflation and overdispersion from two sources: occurrence and abundance of species and occurrence and abundance of predation traces. Though developed in a paleoecological context, this approach is applicable to the study of any predator-prey system in which counts of prey individuals and frequency of predation are each sampled. For example, if it was relevant to the hypothesis they were addressing, the approach presented here could be applied to the data from Blasco-Moreno et al. (2019) to model counts of flowering heads and counts of herbivory traces, allowing for the possibility of Senecio plants without flowering heads.

Count Data in Paleoecological Predation Studies.-Studies of predation frequency in paleoecology are subject to a different set of assumptions and conditions than ecological studies (Table 2). By examining accumulations of dead remains, paleoecologists view the end result of the processes they are studying, which have typically played out over decades, centuries, millennia, or longer. With this expanded temporal perspective come two additional sources of variation that must be accounted for by paleoecologists: preservational bias and time averaging (e.g., Fig. 1). Independently, and in association with each other, the effects of preservational bias and time averaging have been the topic of many studies (e.g., Kidwell and Bosence 1991; Flessa et al. 1993; Kowalewski et al. 1994, 1998; Roy et al. 1994; Kidwell 2001, 2007, 2013; Goodwin 


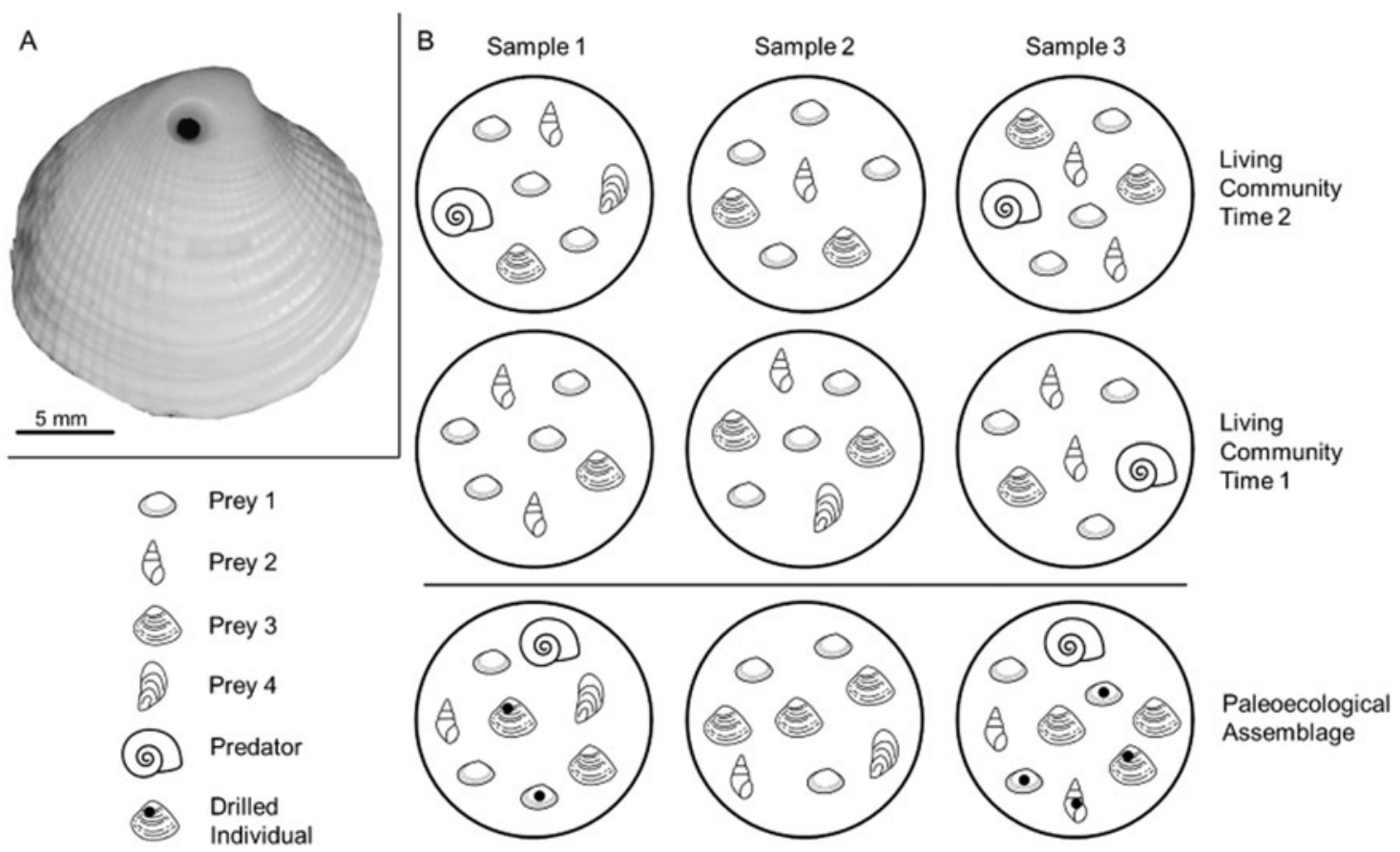

FIGURE 1. A, Example of a drilling trace made by a naticid predator in its clam prey (personal specimen, J.A.S.). B, An illustrative molluscan community, with three samples taken at two points in time, and the hypothetical time-averaged paleoecological assemblage. The predator is present in sample 3 during time 1 and in samples 1 and 3 during time 2; it is found in the paleoecological assemblage in samples 1 and 3 . Time averaging obscures the absence of the predator in sample 1 during time 1. If paleoecological predation frequencies are calculated by pooling data from all samples-including sample 2, where the predator was never present - they would be underestimated because of zero inflation. Counts of individuals are also affected. For example, prey 4 is rare in the living communities but is well preserved in the paleoecological assemblage. With poor preservation, the true abundance of other taxa (e.g., prey 1) is underrepresented in paleoecological samples relative to the living samples. Mollusk drawings from thenounproject.com, with contributors in parentheses: prey 1 (public domain), prey 2 (icon 54), prey 3 (Ker’is), prey 4 (Yu luck), and predator (Juraj Sedlák).

et al. 2004; Behrensmeyer et al. 2005; Olszewski and Kidwell 2007; Klompmaker 2009; Kosnik et al. 2009; Tomašovỳch and Kidwell 2010; Olszewski 2012; Bürkli and Wilson 2017; Dyer et al. 2018; Smith et al. 2019; Sarkar et al. 2020). The consequences of these factors have not been considered, however, with respect to overdispersion and zero inflation as related to paleoecological predator-prey interactions (for a review of existing methods used in paleoecological studies of predation, see Klompmaker et al. [2019]). We focus here on predator-prey interactions in preserved assemblages of recently dead individuals accumulated over the past decade, century, or millennium in the uppermost part of the sedimentary record (i.e., death assemblages; Kidwell 2009).

Preservational bias describes the unequal likelihoods that different individuals, or species, will be preserved in a death assemblage based on a variety of factors, which can include body size, habitat, morphology, scavenging, sedimentation rate, or skeletal composition, to name a few. For example, molluscan individuals with small, thin shells may be more likely to be destroyed than individuals with larger, thicker shells. This bias has the potential to prevent individuals, or species, from being preserved in a death assemblage, which can contribute to zero inflation and, thereby, overdispersion. In many cases, a species may not be entirely removed from the record but will instead be represented by fewer individuals. Depending on the sampling strategy employed, these reduced abundances can also generate zeros in samples, particularly when the species was initially rare or has become rare in the assemblage due to 
preservational bias. Despite this potential bias, death assemblages have been shown to maintain high fidelity to the living assemblages from which they formed, at least with respect to taxonomic composition and rank abundance (e.g., Davis 1923; Johnson 1965; Warme 1969; Kidwell 2001, 2007). With this fidelity in mind, preservational bias can be evaluated as an alternative explanation for observed biotic patterns before drawing conclusions about the pattern or process of interest (e.g., Klompmaker 2009; Sime and Kelley 2016; Smith and Dietl 2016; Johnson et al. 2017; Smith et al. 2019).

Time averaging describes the accumulation of individuals in an assemblage that lived at different times (e.g., Fig. 1B). Unlike most ecological studies, in which any given individual is known to have lived alongside or interacted with the other individuals in its habitat, individuals in a paleoecological study may have lived decades, centuries, or millennia apart. Timeaveraged assemblages tend to have greater richness than the living communities from which they formed because the extended temporal sampling is more likely to capture rare taxa than a survey (i.e., "snapshot") of a living community (Kidwell 2002, 2013; Olszewski and Kidwell 2007; Bürkli and Wilson 2017) and shifts in populations and habitats over time lead to what is essentially extended spatial sampling (Adler et al. 2005; Tomašovỳch and Kidwell 2009). As such, time averaging in a paleoecological dataset may mask a subset of zeros for species occurrences in a community that would be observed in an ecological dataset, if it were possible to concurrently evaluate a dataset from both perspectives. By virtue of the extended sampling, a time-averaged assemblage can be thought of as the average community from the period over which the assemblage accumulated. Yet, as the averaging smooths out temporal variation in the data, muting shortterm trends and patterns (Kowalewski et al. 1998), it introduces additional uncertainty and variability with respect to sampling effort. In addition to the inherent uncertainty in estimates of time averaging, variability among samples and among species in the degree of time averaging (e.g., Kosnik et al. 2009; Kowalewski et al. 2018) unavoidably increases uncertainty in the data, particularly if samples are from more than one locality or time period. Thus, analytically, there is, or at least should be, a cost associated with time-averaged data. To address this uncertainty, paleoecologists typically assume accumulation of specimens in an assemblage occurred at a constant rate and the degree of time averaging is the same across samples, though neither is likely to hold (Holland 2016; Tomašovỳch et al. 2016; Hopkins et al. 2018).

The effects of preservational bias and time averaging also apply to counts of predation traces, such as drill holes (for a review of these effects see Klompmaker et al. [2019]). In marine habitats, drill holes are often the result of predation by snails (e.g., Fig. 1A), commonly of the families Muricidae and Naticidae, on other snails and clams. The presence of a drill hole in a shell is taken as evidence that the drilled individual died as a result of predation. In studies of drilling predation, the frequencies upon which prey types - used generically here to refer to genera, species, morphospecies, size groups, or any other grouping of individualsare drilled (i.e., consumed) are used to evaluate hypotheses relating to both ecology (e.g., prey selection; Kitchell et al. 1981; Dietl and Alexander 1995; Leighton 2003; Chattopadhyay and Dutta 2013; Smith et al. 2018a) and evolution (e.g., coevolution and escalation; Vermeij 1987; Kelley 1989, 1991; Dietl and Alexander 2000; Kelley and Hansen 2003; Mallick et al. 2014; Harper 2016; Klompmaker et al. 2017).

The effects of time averaging on drill hole counts are similar to those for specimen counts; however, drill holes introduce an additional element on which preservational bias can act. Shells with drill holes may be more susceptible to breakage compared with their undrilled counterparts (e.g., Roy et al. 1994; Sarkar et al. 2020), though other studies have found a negligible effect of drill holes on preservational potential (e.g., Kelley 2008; Dyer et al. 2018). The effects of drill holes on shell strength and preservation remain a topic of debate and are likely to vary by prey type, based on other underlying characteristics of the shell (e.g., size, thickness) and depositional environment (e.g., sedimentation rate, wave-energy exposure). If there is an effect, which is very likely for some prey types, it has the potential to 
contribute to zero inflation in predation count data by removing drill holes from the dataset (Table 1). Given the extent of variability in this effect in terms of prey type and depositional setting, we focus on overdispersion introduced by time averaging and the general process of modeling zero inflation in paleoecological drilling predation studies.

\section{Modeling of Species Abundances and Predation Events}

Ecological studies accounting for zero inflation and overdispersion typically consider their effects on a single source of count data (e.g., individuals or predation traces). For example, although Blasco-Moreno et al. (2019) used per-plant counts of flowering heads available to herbivores as an explanatory parameter, counts of flowering heads were not modeled in the same fashion as counts of predation (i.e., herbivory) traces, reflecting the hypothesis they were testing (i.e., zero counts for number of flowering heads would have precluded testing the enemy release hypothesis). The hierarchical Bayesian model we present here includes the potential to model the counts of individuals of different prey types in samples from an assemblage and counts of predation traces on each prey type, while accounting for overdispersion and zero inflation.

Modeling Species Abundances. - Count data in studies of paleoecological predator-prey interactions often take the form $\left(x_{i}, y_{i}\right)$, where $y_{i}$ is the number of specimens and $x_{i}$ is the number of predation events in sample $i$ of $N$ total samples. In general, sample sizes $\left(y_{i}\right)$ are random, and the canonical sampling process is a Poisson process. Yet, in real datasets, counts $\left(y_{i}\right)$ rarely obey a simple Poisson model (Hougaard et al. 1997). Instead, counts are often overdispersed, potentially due to an excess number of zeros. Similarly, counts of predation events $\left(x_{i}\right)$ can be zero-inflated. To draw ecological inferences from such samples, a fundamental question must be addressed: Are these deviations from the Poisson model a result of sampling issues or actual ecological conditions?

We first address this question with respect to sample counts and subsequently apply the developed hierarchical Bayesian framework to a case study. This approach does not obviate traditional maximum likelihood methods (Cameron and Trivedi 2013), which in some applications can provide adequate models. The Bayesian framework allows for more flexible and faithful models, because it better captures sources of variations and the hierarchical (i.e., multilevel) nature of the phenomenon (Gelman and Hill 2006; Kéry 2010; KornerNievergelt et al. 2015; van de Schoot et al. 2021). This approach is particularly useful when data deviate from the ideal Poisson sampling model and violate the assumption that samples represent the same underlying abundance and sampling effort. We consider first the scenario wherein the spatial distribution of specimens is patchy or aggregated owing to a depositional process (e.g., size sorting, winnowing) or an underlying ecological phenomenon. Sample counts would then likely exhibit overdispersion, because the variance of the counts would exceed the mean of the count values. From a Bayesian perspective, this additional variance can be incorporated in a hierarchical model in which the parameter of the Poisson distribution is itself subject to randomness,

$$
\begin{aligned}
& y_{i} \sim \operatorname{Poisson}(\lambda) \\
& \lambda \sim \operatorname{Gamma}(\alpha, \beta)
\end{aligned}
$$

When applying a gamma prior to estimate the Poisson mean, $\lambda$, the hierarchical model is equivalent to a negative binomial distribution. There are many reasonable choices for the distribution of $\lambda$ (e.g., Student's $t$ ), which have thick tails (Hougaard et al. 1997). However, the gamma distribution is a very flexible distribution capable of taking on many shapes and serves as a versatile model for variation in $\lambda$. In addition, the Bayesian approach does not require closed-form formulas for the posterior distribution (e.g., negative binomial). Priors allow for greater probabilities of large outcomes, potentially arising from preservational bias and time averaging. This approach captures the reality that the underlying abundances of each taxon have their own unique distributions and that variation is explained by the probability distribution of $\lambda$.

In addition to the underlying variation in the distributions of individual taxon abundances, 
differences in sampling effort, including the degree of time averaging, can also generate variation. The actual sampling effort represented in a sample is difficult to assess (Olszewski 2012; Holland 2016; Tomašovỳch et al. 2016). Among the many processes involved are (1) the "influx" process, which accounts for the biological generation of the specimens themselves (i.e., the underlying abundance) and can vary over time (e.g., Olszewski 2012); (2) the processes by which specimens are removed through decay, erosion, scavenging, and other processes (e.g., Olszewski 2012; Tomašovỳch et al. 2016); and (3) specimen accumulation over time (i.e., time averaging), which can be variable. For example, estimates of time in assemblages from spatially proximal sediment cores can differ by thousands of years (Kosnik et al. 2009). These effects are rarely, if ever, quantified in paleoecological studies of predator-prey interactions. Alternatively, variation imparted by these processes can be captured in the model by utilizing more flexible distributions. For instance, if a spatial covariate, like sampling area, were available, variation in the mean could be modeled as,

$$
\begin{aligned}
y_{i} & \sim \operatorname{Poisson}\left(\lambda_{i}\right) \\
\lambda_{i} & \sim \operatorname{Gamma}\left(\alpha_{i}, \beta\right) \\
\alpha_{i} & =b * \operatorname{area}_{i}
\end{aligned}
$$

Going a step further, the time represented in a sample (b) can be modeled to account for uncertainty associated with differences in the degree of time averaging among samples,

$$
\begin{aligned}
y_{i} & \sim \operatorname{Poisson}\left(\lambda_{i}\right) \\
\lambda_{i} & \sim \operatorname{Gamma}\left(\alpha_{i}, \beta\right) \\
\alpha_{i} & =b * \operatorname{area}_{i} \\
b & \sim \operatorname{Lognormal}(\log \mu, \log \sigma)
\end{aligned}
$$

After accounting for this potential underlying variability across samples, the next issue to address is the excess of zeros in the dataset relative to the Poisson sampling process and to determine their origin (Table 1). These zeros can be addressed with a zero-inflated model, allowing zeros from ecological and sampling processes (e.g., Martin et al. 2005; Wenger and
Freeman 2008; Blasco-Moreno et al. 2019). Such a model is fundamentally a mixture model that allows for a zero to be structural (i.e., ecological), with a certain probability $\left(\omega_{\mathrm{s}}\right)$, or a sampling zero, with one minus that probability,

$$
g\left(y_{i} ; \lambda, \omega_{\mathrm{s}}\right)=\omega_{\mathrm{s}} I\left(y_{i}=0\right)+\left(1-\omega_{\mathrm{s}}\right) f\left(y_{i} ; \lambda\right)
$$

where $I$ is an indicator function with a value of one if the condition in the parentheses is true and zero otherwise. The distribution, $f$, in the second term could be the idealized Poisson distribution or another sampling distribution, including any of the hierarchical types previously described (e.g., eq. 3). With this hierarchical Bayesian approach, sources of uncertainty inherent to paleoecological (and ecological) count data can be better incorporated into ecological inferences.

Modeling Predation Events.-As previously described, predation data comprise two counts, $\left(x_{i}, y_{i}\right), i=1, \ldots, N$, where $y_{i}$ is the number of specimens and $x_{i}$ is the number of predation events in sample $i$. The number of specimens, $y_{i}$, can be estimated by Bayesian methods as described in the preceding section. In an idealized setting, counts of predation events $\left(x_{i}\right)$ can be estimated as a binomial distribution, where $x_{i}$ are "successes" in $y_{i}$ potential encounters between predator and prey, with a probability of success, $p$. Parameter $p$ is the predation frequency (e.g., drilling frequency: the number of drilled individuals divided by the sum of drilled and undrilled individuals). This idealized case arises when predatory events are independent of each other and can be written as,

$$
\begin{aligned}
x_{i} & \sim \operatorname{Binomial}\left(p, y_{i}\right) \\
y_{i} & \sim f\left(\lambda_{i}\right)
\end{aligned}
$$

where the sampling distribution, $f$, is the idealistic Poisson distribution or, to better account for variation and uncertainty, another count distribution, such as the hierarchical distributions previously described in equation 3 . In this case, we assume predation probability is constant in space and time, but that, too, is unrealistic. For example, the predation probability may be a function of predator abundance (e.g., Hansen and Kelley 1995; 
Chiba and Sato 2014; Stafford et al. 2015) or biased by differential preservation probabilities (e.g., Walker 1989; Klompmaker 2009; Smith et al. 2019). At the risk of over-parameterizing, we can conceptually elaborate this model to include sample-to-sample variation, as we did with sample counts (e.g., eq. 1), by introducing a distribution on predation probabilities,

$$
\begin{aligned}
x_{i} & \sim \operatorname{Binomial}\left(p_{i}, y_{i}\right) \\
y_{i} & \sim f\left(\lambda_{i}\right) \\
p_{i} & \sim h\left(\theta_{i}\right)
\end{aligned}
$$

where $h$ is an appropriate distribution such as beta, which is a standard model for random values between 0 and 1 . Moreover, if each sample had a covariate, $z_{i}$, which potentially influences predation counts (e.g., abundance of predators, preservational bias, water depth), it could be incorporated via logistic regression (Wenger and Freeman 2008),

$$
\begin{aligned}
& x_{i} \sim \operatorname{Binomial}\left(p_{i}, y_{i}\right) \\
& y_{i} \sim f\left(\lambda_{i}\right) \\
& \operatorname{logit}\left(p_{i}\right)=\beta_{0}+\beta_{1} z_{i}
\end{aligned}
$$

Though data on preservational bias are not assessed in our case study, such data could be incorporated here as a covariate via logistic regression.

It is possible that counts of predation events $\left(x_{i}\right)$ are zero-inflated resulting from, for instance, the absence of predators from a habitat due to patchiness during the period over which the sample accumulated, avoidance of certain prey types by the predator, or preservational bias (Table 1). Thus, even when counts of specimens $\left(y_{i}\right)$ are positive, it is possible that no predation would have occurred (i.e., $x_{i}=0$ ). It is also possible that a zero is a random sampling artifact, resulting from a small sample size $\left(y_{i}\right)$ relative to predation frequency, $p$ (Table 1). As with modeling sample counts, a mixture model captures the probability of excess zeros,

$$
\begin{aligned}
h\left(x, y ; p, \omega_{p}\right)= & \omega_{p} I(x=0) \\
& +\left(1-\omega_{p}\right) \operatorname{Binomial}(x ; p, y)
\end{aligned}
$$

Allowing counts of predation events (i.e., drill holes) to be zero-inflated enables more precise estimates of the true predation frequency. When estimating predation frequency, the intent is to measure the frequency of attacks when the prey type is "on the menu" and predators are possibly present (i.e., there is a nonzero probability the predatory interaction did or could have occurred). Including all zeros in counts of predation traces from all samples can lead to underestimates of predation frequency. Thus, a zero-inflated model reduces potential downward bias in predation frequency estimation by using a mixture model to differentiate between zeros generated by the phenomenon of interest (i.e., predation) and those originating from other factors (e.g., sampling).

One final component to consider probabilistically is the scenario in which observed specimen and predation event counts are both zero (i.e., $x_{i}=0, y_{i}=0$ ). This scenario can arise in several ways, including when (1) a prey type count is a false zero (e.g., specimens existed but were not sampled due to preservational bias) and predators were present (i.e., $x_{i}=0$ is a sampling artifact); (2) a prey type count is a false zero and predators were not present to drill specimens (i.e., $x_{i}=0$ is structural, with an ecological explanation); or (3) a prey type count is a true zero (i.e., ecological) in the sense that specimens were not available for sampling and the predation event count is necessarily zero. For the sake of simplicity, we treat these three possibilities together, with case 1 , below. Two alternatives can be applied to estimate predation frequency when specimens are present $\left(y_{i}>0\right)$, without predation (case 2$)$ or with predation (case 3):

1. $\left(x_{i}=0, y_{i}=0\right): \omega_{s} I\left(y_{i}=0\right)+\left(1-\omega_{s}\right) f(0 ; \lambda)$

2. $\left(x_{i}=0, y_{i}>0\right)$ :

$\omega_{p} I(x=0)+$

$\left(1-\omega_{p}\right) \operatorname{Binomial}\left(0 ; p, y_{i}\right) ; y_{i} \sim f(y ; \lambda)$

3. $\left(x_{i}>0, y_{i}>0\right)$ :

$\operatorname{Binomial}\left(x_{i} ; p, y_{i}\right) ; y_{i} \sim f(y ; \lambda)$

In these three cases, the distribution $f$ could be the Poisson distribution or any other sampling distribution (eq. 4). As demonstrated above, the model comprising these three cases can become a hierarchical model by allowing parameters $\lambda$ and $p$ to have indices that vary by sample 
according to their own distributions (eqs. 1 and 6). Allowing sample-to-sample variation, as facilitated by the hierarchical Bayesian framework, can better account for the uncertainty inherent to ecological and paleoecological datasets and can improve the inferences drawn from them.

Model Evaluation.-These hierarchical models can all be fit within the Bayesian paradigm using a probabilistic programming language (e.g., Stan; Stan Development Team 2019). As noted elsewhere, hierarchical Bayesian models, especially as implemented through probabilistic programming languages such as BUGS or Stan, provide flexibility to free the modeler without regard to estimation details (Kéry 2010; Korner-Nievergelt et al. 2015). In the approach facilitated by sampling methods for posterior distribution estimates, we have the ability to produce true probability statements about the values of parameters, given the data and the model.

One limitation of the sampling approach is the uncertain nature of model ranking. Although many researchers rely on the Akaike information criterion (AIC) to rank scientifically plausible and well-fitting models, the current situation in sampling-based Bayesian estimation is that it is often difficult to select the best model from a set of candidates owing to approximations and estimation error, especially for complicated hierarchical models (see Gelman and Hill 2006). Model-ranking methods like AIC break down for hierarchical models, because counting the number of parameters is not straightforward. Consequently, similar methods (e.g., the deviance information criterion) have been developed for the Bayesian framework to be used in place of AIC; however, because of their inherent approximations, they have difficulty evaluating competing models (Gelman and Hill 2006). It is thus generally advised to perform predictive model checking (Gelman and Hill 2006; Korner-Nievergelt et al. 2015) to assess model fit and to rely heavily on scientific insight and interpretability for model ranking (Vehtari et al. 2017). Posterior predictive checking simulates the fidelity of the model and its fit to the data by drawing random model parameters from their posterior distributions and then simulating data from that random model. If the model fits well, the actual data and simulated data should generate similar histograms and summary statistics. This method is sometimes used as a companion to other model diagnostics (e.g., information theoretic criterion), which may not be informative when sample sizes are too small for the symptotic formulas to hold (Korner-Nievergelt 2015). We follow this approach to model checking (see Supplementary Material).

\section{A Paleoecological Case Study}

To illustrate the potential effects of overdispersion and zero inflation in paleoecological studies, we analyzed data from Martinelli et al. (2015) using the framework developed in the preceding section. We did not reevaluate the hypotheses presented in Martinelli et al. (2015). Instead, we used their data to highlight sources of variation that may confound many, if not all, paleoecological studies using count data and to illustrate a statistical framework to address these sources of variation. All analyses used the Bayesian programming language Stan (Stan Development Team 2019) called from within the $\mathrm{R}$ statistical programming environment using the package rstan (Stan Development Team 2018). All code and data are available in the Supplementary Material.

The data from Martinelli et al. (2015) are from replicate samples of the molluscan living and death assemblages in three lagoons at One Tree Reef, in the southern Great Barrier Reef. Fifteen sampling sites were included, with seven from the first lagoon and four from each of the others. Four replicates were collected at each site from a $0.25 \mathrm{~m} \times 0.25 \mathrm{~m}$ area on the seafloor, to a sediment depth of $10 \mathrm{~cm}$. Sampling was repeated four times throughout the year, resulting in 16 samples from each site. We only assessed the death assemblage samples here-one sample from each site was processed in most cases, because death assemblages often contain more individuals than the living assemblage. When death assemblages contained relatively few individuals, additional samples were included, resulting in different sampling efforts for some sites (see Supplementary Table 1). All processed samples 
were sieved on a 4-mm mesh, and all individuals were identified to the species level. Occurrences of predatory drill holes were also recorded, and predation frequencies were calculated.

As is the case for most paleoecological studies, including our own work, Martinelli et al.'s (2015) original study did not assess the degree to which the death assemblages were time-averaged, which was a source of variation of interest in our analysis. To remedy this lack of temporal context for our illustrative case study, we applied time-averaging data from Kosnik et al. (2009), which were collected from molluscan death assemblages at Rib Reef on the Great Barrier Reef. The data from Kosnik et al. (2009) are from two 125-cm-deep cores of the seafloor sediments. In the top $25 \mathrm{~cm}$ of the cores, shells were typically younger (i.e., from the last several years or decades) than those from the deeper part of the core, and from 25 to $125 \mathrm{~cm}$, shell ages were well mixed throughout. Time averaging was on the scale of thousands of years, but varied considerably between cores (e.g., ages for Abranda casta ranged from 2 to 1019 years in core 1 compared with 4 to 4670 years in core 2; see Supplementary Fig. 1).

We acknowledge these two datasets are from disparate locations on the Great Barrier Reef and may not truly hold any bearing on each other. Ideally, time-averaging data should be collected from the same samples being analyzed to evaluate (paleo)ecological hypotheses; however, there is a paucity of such studies. Thus, because our objective was to develop an analytical framework with the capacity to incorporate data on time averaging and illustrate the potential biasing effects of overdispersion and zero inflation, we proceeded with this combined dataset for illustrative purposes.

Overdispersion Analysis.-In the data from Martinelli et al. (2015), specimen counts varied greatly for many species (e.g., 19-925, Pinguitellina robusta; see Supplementary Table 1). This variance resulted in overdispersion, as noted by the authors: "the ratio of model residual deviances to residual degrees of freedom tended to be greater than one, indicating overdispersion" (p. 820). The counts for P. robusta are representative of those common in death assemblage samples and were used here to illustrate the presence of overdispersion and incorporation of greater uncertainty into a paleoecological model.

Sample-to-sample differences in counts likely arose from ecological processes (e.g., patchiness, aggregation) due to underlying environmental conditions (e.g., water depth, temperature) or variation in sampling effort. We fit four models to these count data and evaluated goodness of fit using a posterior predictive check (Table 3). Without information on environmental conditions (i.e., a covariate), any differences are effectively random. To account for this randomness, we allowed the mean count to vary more than permitted in a simple Poisson distribution by including a gamma prior (i.e., eq. 1):

$$
\begin{aligned}
& y_{i} \sim \operatorname{Poisson}(\lambda) \\
& \lambda \sim \operatorname{Gamma}(\alpha, \beta)
\end{aligned}
$$

Alternatively, differences in sampling effort owing to sampling area and time averaging can be incorporated. First adding sampling area, we fit a model in which the area is presumed to be the main driver of specimen counts, while also allowing for extra variation from a gamma distribution:

$$
\begin{aligned}
y_{i} & \sim \operatorname{Poisson}\left(\lambda_{i}\right) \\
\lambda_{i} & \sim \operatorname{Gamma}\left(\alpha_{i}, \beta\right) \\
\alpha_{i} & =b * \text { area }_{i}
\end{aligned}
$$

Because the mean of the $\operatorname{Gamma}\left(\alpha_{i}, \beta\right)$ distribution is $\alpha_{i} / \beta$, estimates of $\alpha_{i}$ and $\beta$ will track expected counts through $\lambda_{i}$.

To this point, the analysis could apply equally to ecological or paleoecological data. As the Martinelli et al. (2015) data are paleoecological, it is also reasonable to assume some degree of time averaging is contributing to the variation in the counts, which, in the absence of data from Martinelli et al.'s samples, we drew from Kosnik et al. (2009). As discussed above, the accumulation of a death assemblage is complicated and includes random influx of individuals, a suite of temporally variable processes that remove individuals, and right-censorship of the data (Tomašových et al. 2016). Without auxiliary data on accumulation, we could not infer from first principles how specimens actually accumulated in the sample. Consequently, 
TABLE 3. Parameter estimates for the posterior predictive check assessing goodness of fit for models of counts for Pinguitellina robusta. Estimates for each parameter of the various models are given as the posterior mean, with associated standard deviation (SD) and 95\% credibility regions $(2.5 \%-97.5 \%)$.

\begin{tabular}{|c|c|c|c|c|c|}
\hline Model & Parameter & Posterior mean & SD & $2.5 \%$ & $97.5 \%$ \\
\hline Poisson & Lambda & 274.73 & 4.24 & 266.49 & 283.28 \\
\hline \multirow[t]{3}{*}{ Poisson-gamma } & Lambda & 274.81 & 4.32 & 266.48 & 283.56 \\
\hline & Alpha & 6.64 & 2.44 & 1.39 & 9.92 \\
\hline & Beta & 0.03 & 0.01 & 0.01 & 0.06 \\
\hline \multirow[t]{3}{*}{ Poisson-gamma-area } & Lambda & 273.54 & 4.37 & 264.68 & 282.19 \\
\hline & B1 & 3.23 & 1.00 & 1.57 & 5.50 \\
\hline & Beta & 0.01 & 0.01 & 0.01 & 0.02 \\
\hline \multirow[t]{4}{*}{ Poisson-gamma-area-time (lognormal) } & Lambda & 272.69 & 3.79 & 265.16 & 280.57 \\
\hline & B1 & 4.13 & 10.03 & 0.32 & 35.80 \\
\hline & Time & 3.18 & 2.81 & 0.10 & 11.98 \\
\hline & Beta & 0.02 & 0.01 & 0.01 & 0.02 \\
\hline \multirow[t]{3}{*}{ Poisson-lognormal } & Lambda & 274.85 & 4.24 & 266.32 & 282.93 \\
\hline & Log_mu & 3.99 & 2.34 & 0.16 & 7.88 \\
\hline & Log_sigma & 3.11 & 6.01 & 0.32 & 13.60 \\
\hline
\end{tabular}

we applied the assumption that time averaging is positively correlated to specimen counts (Tomašovỳch and Kidwell 2010). By extension, we necessarily also assumed that rates of specimen accumulation exceeded rates of specimen removal and that both rates were relatively constant over the period of time averaging (Table 2). Reflecting the uncertainty associated with these assumptions, we used a general, flexible model to capture variation in sampling effort.

To incorporate the uncertainty introduced by time averaging into the model, we fit a lognormal distribution to the time-averaging data for A. casta-formerly known as Tellina casta; a confamilial of $P$. robusta-from two cores sampled by Kosnik et al. (2009; see Supplement 1). We used the parameters of this fitted lognormal, $\hat{\mu}=6.64, \hat{\sigma}=1.99$, as a prior to simulate average accumulation time-interval lengths in the model,

$$
\begin{aligned}
& y_{i} \sim \operatorname{Poisson}(\lambda) \\
& \lambda \sim \operatorname{Gamma}\left(\alpha_{i}, \beta\right) \\
& \alpha_{i}=b * \operatorname{area}_{i} * \text { time } \\
& \text { time } \sim \operatorname{Lognormal}(6.64,1.99)
\end{aligned}
$$

As with the previous model, the mean of the $\operatorname{Gamma}\left(\alpha_{i}, \beta\right)$ distribution is $\alpha_{i} / \beta$, such that estimates of $\alpha_{i}$ and $\beta$ track the expected counts through $\lambda_{i}$. In essence, the model implies sampling area and accumulation time drive the count process, with extra variation captured by allowing each sample its own gamma distribution. Because there are no environmental covariates in the model, this last model assumes original abundances were constant across sampling areas.

We used posterior predictive model checking to evaluate the models (Table 3 ). The variation in sample sizes was not adequately explained by the Poisson or Poisson gamma models. The model using sampling area as a covariate fared better, because area increased with sample size, but still not enough to capture the larger sample sizes. The model with a lognormal prior did not offer much improvement over the area covariate model owing to the significant amount of count data that overwhelmed the prior.

In the absence of covariates that more completely capture sampling effort, of which time averaging is a critical component, it is difficult to fully model the sampling process and expose the underlying abundance. The plausible lognormal fit to the age data from Kosnik et al. (2009) suggests time averaging could be an important driver of sample-size variation. That is, time-averaging data collected from the same specimens analyzed for predatorprey interactions may better explain the variance and improve the model fit. Likewise, adding data on alternative explanatory variables (e.g., environmental factors, preservational bias) as covariates (e.g., eq. 7) would reduce the reliance on assumptions (e.g., Table 2) and likely improve model fits. Data issues notwithstanding, the framework developed here 
allows for the incorporation of accumulation processes into estimates of specimen counts, representing a step forward in paleoecological analytical methods.

Zero-inflated Predation Frequencies.-In paleoecological studies, drilling predation frequencies are typically calculated by pooling the number of drilled and undrilled individuals across samples and dividing the total number of drilled individuals by the total number of individuals. Intuitively, when the pooled number of individuals is high the estimate of predation frequency can be interpreted more confidently and when the pooled number of individuals is low (e.g., $n<30$ ) there is greater uncertainty in the estimate. The uncertainty is increased when the number of individuals in each unique sample is low. It is this latter case we evaluated here, using the snail Notocochlis gualtieriana from the Martinelli et al. (2015) study as an example (see Fig. 2 for analysis of species with at least 15 total individuals; see also Supplementary Fig. 2).

In 5 of the 15 sampled sites, no N. gualtieriana specimens were reported. Specimen counts ranged from 1 to 12 in the 10 sample sites where $N$. gualtieriana was found. In total, only one $N$. gualtieriana specimen was drilled, indicating that the predatory interaction was ecologically plausible (see Supplementary Table 2). Pooled together, N. gualtieriana was represented by 43 individuals and, following the traditional calculation, was drilled with a frequency of 0.02 . This traditional approach does not address the abundance of zeros in the drilling data; in 9 of 10 samples, no N. gualtieriana specimens were drilled. Thus, we fit a zero-inflated mixture model to the data. As the dataset is small and apt to support a variety of models, we fit a single, ecologically plausible model and evaluated it with posterior predictive checking: (i.e., eq. 8)

$$
\begin{aligned}
h\left(x, y ; p, \omega_{p}\right)= & \omega_{p} I(x=0) \\
& +\left(1-\omega_{p}\right) \operatorname{Binomial}(x ; p, y)
\end{aligned}
$$

In this case, the model fit was good, as the posterior predictive check indicated the actual and simulated data had similar histograms and summary statistics (Table 4). With the zero-inflated model, the predation frequency $(p)$ was estimated to be 0.24 , indicating considerable underestimation with the traditional approach. The difference is largely the result of allowing for the second zero-generation process in the model. Indeed, a majority of the zeros in the dataset for N. gualtierianna were determined to be true, structural zeros (i.e., the absence of predation had a probable ecological explanation).

Considering all species in the death assemblage with at least 15 individuals (Fig. 2), we found that predation frequencies for several other species (e.g., Atys hyalina, Tellina gargadia) were also underestimated due to zero inflation. In general, species can be put into three groups: high abundances and few ecological zeros (left side of figure); moderate pooled abundances but low abundances in each sample and many ecological zeros (right side of figure); and species with low pooled abundances precluding meaningful inference (not shown in Fig. 2; see Supplementary Fig. 2). The differences between estimates of predation frequencies calculated with the traditional approach and with a zero-inflated model are most pronounced in the middle group. Ecologically, this is plausible when considering the distribution and abundance structure of species in communities. Often, a subset of species in the community will be highly abundant and these species will be heavily preyed upon, numerically, as a function of their abundance. Even so, those abundant species may rank low in a predator's preference hierarchy, and less common taxa may exhibit greater predation frequencies per capita in places and times when the predator and prey overlap (Kitchell et al. 1981; Hansen and Kelley 1995; Martinelli et al. 2015; Smith et al. 2018b). Predation of this second variety is better represented with a zero-inflated model than with the traditional approach applied in paleoecology.

\section{Discussion}

On the surface, paleoecological and ecological count data appear to be nearly identical. Many, if not all, metrics applied to ecological count data can also be applied to paleoecological count data, and both types of data are susceptible to zero inflation and 


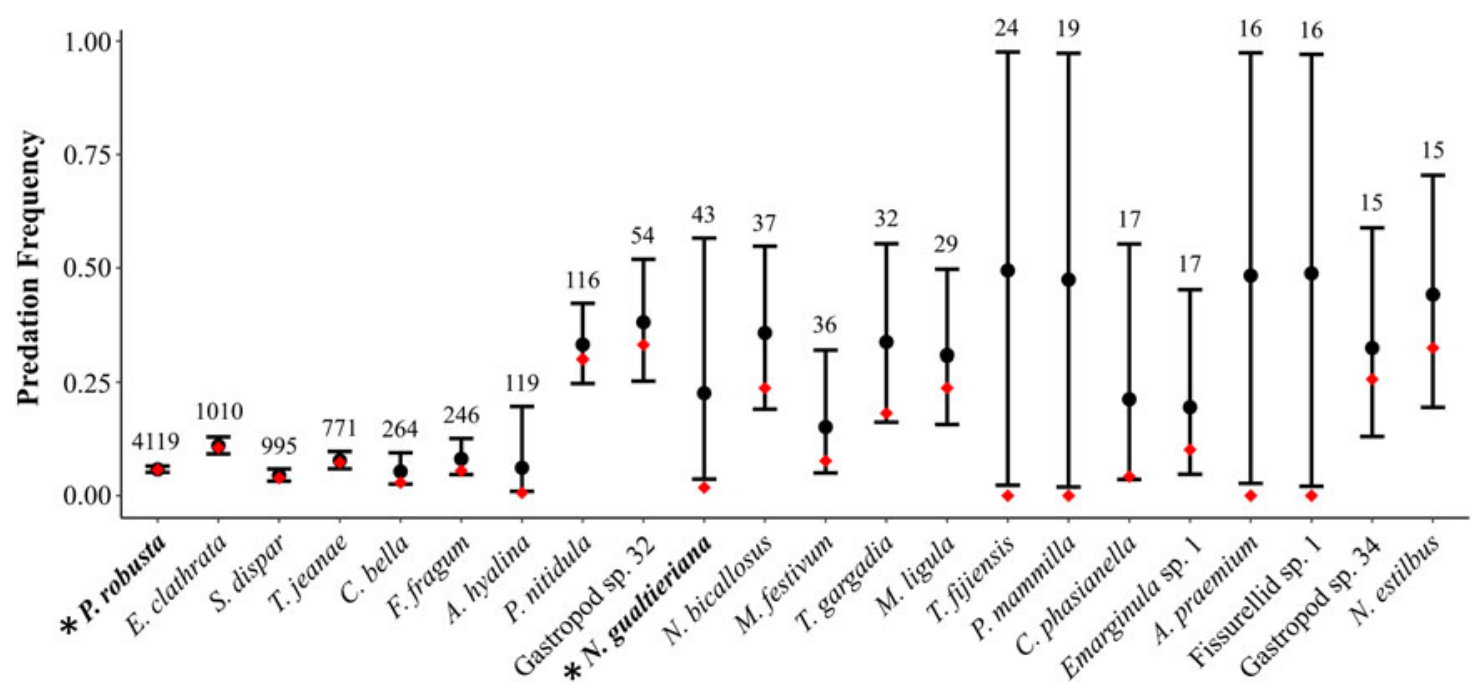

FIGURE 2. The effect of zero inflation on estimates of predation frequency. Species are sorted by sample size in decreasing order from left to right, with specimen counts given above the black bars for confidence regions - species with fewer than 15 individuals were excluded (see Supplementary Fig. S2). Black circles represent predation frequencies accounting for zero inflation $(p)$, with 95\% confidence regions (black bars). Red diamonds represent predation frequencies for each species estimated with the traditional calculation (number of drilled individuals divided by the total number individuals in each sample). Data from Martinelli et al. (2015). Asterisks on the x-axis with bolded taxon names indicate the two species, Pinguitellina robusta (far left) and Notocochlis gualtieriana (central), discussed in the main text.

overdispersion. Yet, because of preservational bias and time averaging, paleoecological count data are inherently more variable and carry a greater degree of uncertainty than ecological count data (Fig. 1B). In paleoecological studies, these effects are often addressed by making assumptions (Table 2) or assessed as alternative explanations for observed biological patterns. That is, they are either ignored a priori or discounted after the fact-including in our own previous work. The hierarchical Bayesian framework developed here allows for the effects of time averaging and preservational bias to be included while examining

TABLE 4. Parameter estimates for the posterior predictive check assessing goodness of fit for the model of predation counts on Notocochlis gualtieriana. Predation frequency $(p)$ is estimated using the hierarchical Bayesian model. For comparison, the predation frequency estimate using the traditional method (number of drilled individuals divided by the total number of individuals) is 0.02 . SD, standard deviation.

\begin{tabular}{lcccc}
\hline \hline Parameter & Posterior mean & SD & $2.5 \%$ & $97.5 \%$ \\
\hline Omega_predation & 0.82 & 0.11 & 0.56 & 0.98 \\
Omega_sampling & 0.35 & 0.11 & 0.14 & 0.57 \\
Lambda & 4.48 & 0.65 & 3.29 & 5.86 \\
$p$ & 0.24 & 0.13 & 0.04 & 0.56 \\
\hline
\end{tabular}

potential biological processes and patterns. In essence, the analysis treats variation introduced by preservational bias and time averaging as if it was overlain on the variation inherent to the ecological processes, which is more realistic.

Analytically, incorporation of these sources of variation is important when choosing the model to represent the data. Each model carries assumptions about how the data, and thereby community or assemblage, are structured. As demonstrated in the case study here and in previous studies (Martin et al. 2005; Wenger and Freeman 2008; Blasco-Moreno et al. 2019), whether the underlying assumptions of the models are valid (e.g., variance equal to the mean in a Poisson distribution) can influence the model that best fits the data. Despite the similarities in ecological and paleoecological count data, it is often more challenging to validate these assumptions in paleoecology because of the long-acting processes at play. Consequently, there is more uncertainty in paleoecological inferences. By accounting for uncertainty from time averaging within a hierarchical Bayesian framework, our case study suggests that those inferences can be improved. 
To address this uncertainty with respect to zero inflation and overdispersion, estimation of the potentially confounding sources of variation (i.e., time averaging, preservational bias) is a necessity. Though it was hitherto rare for such data to be collected and integrated into a single paleoecological study, the risk of misinterpreting data and drawing erroneous conclusions makes the incorporation of such data paramount. Whereas the effect of preservational bias will tend to be unidimensional (i.e., removal of specimens resulting in zero inflation), the effects of time averaging are more complicated.

As treated here, time averaging can be thought of as a temporal sampling window. Whether an assemblage accumulated over a decade, century, or longer, the end result is a set of individuals that have been "sampled" from the entire period of time (Fig. 1B). In a dataset composed of such samples, time averaging has two potential effects: (1) reduced zero inflation because of extended temporal sampling capturing rare events and occurrences and (2) increased uncertainty because of sample-to-sample variability in the extent of time averaging. The first effect, capture of rare occurrences, is often viewed as a positive attribute, as it allows for a more complete accounting of the average state of the community. Typically, the "rare occurrence" being captured is that of a rare species in a community (e.g., Prey 4 in Fig. 1B), which results in greater richness of death assemblages (Kidwell 2002, 2013; Olszewski and Kidwell 2007; Bürkli and Wilson 2017). This effect likely can also be extended to rare predation events. For example, if a prey type is ranked low in a predator's preference hierarchy, predation on this prey type may only occur with very low frequencies (e.g., $<1 \%$ ). Yet, because time averaging captures rare occurrences, it would be more likely to find a rare instance of predation in a death assemblage than in a survey of a corresponding living community. Thus, this first effect likely reduces zero inflation, and thereby overdispersion, with respect to the occurrence of species in an assemblage and the incidence of rare predation events.

The second effect, increased sample-tosample variability in extent of time averaging, increases the uncertainty in inferences drawn from the count data. Though it is often assumed, individuals likely do not accumulate in an assemblage at a constant rate, especially individuals of different species (Kosnik et al. 2009; Olszewski 2012; Holland 2016; Tomašovỳch et al. 2016; Kowalewski et al. 2018). Many factors (e.g., bioerosion intensity, sedimentation rate, water chemistry, taxonomy) affect the inclusion of specimens in an assemblage, and they vary through space and time. Ecological communities also vary through space and time, with various correlations to the factors affecting preservation. Consequently, the living community from one year may contribute more individuals to a death assemblage than the living community from the next year or the living community 10 years later. Though paleoecologists do regularly standardize one aspect of sampling effort by taking volumetrically controlled samples (i.e., bulk samples) from a locality, the true sampling effort represented by a paleoecological sample is uncertain, because the assemblage itself accumulated variably. For example, in the two cores used here to estimate time averaging, there was a fourfold difference in the range of ages of individuals - core 1 ranged from 2 to 1019 years and core 2 ranged from 4 to 4670 years - despite samples being taken from the same general area (Kosnik et al. 2009). If one is only comparing samples from the same assemblage, there is a greater likelihood that the temporal sampling effort will be similar; however, when comparing assemblages from different times and places, time averaging may vary substantially (Kowalewski et al. 1998; Kosnik et al. 2009; Ritter et al. 2017). Incorporation of time averaging into analyses of count data can improve model fits by accounting for this additional variability.

The Issue of Preservational Bias.-The assumptions used commonly by paleoecologists to account for the effects of preservational bias are not unlike assumptions applied in ecological studies. For example, in surveys of ecological communities, it is common to assume that all individuals have equal and independent detection probabilities (Martin et al. 2005; Wenger and Freeman 2008). Intuitively, we know that this is unlikely to hold because of variations in size, morphology, behaviors, and life histories, among others. The paleoecological assumption 
that all individuals are equally likely to be preserved in an assemblage, and thus equally likely to be found in a paleoecological sample, is based on the same general sampling principles. Both assumptions rest on the shared premise that the sample taken is representative of the potential individuals that were available (i.e., living in the community) for sampling. Convenient as it is to make this assumption in paleoecological studies, several studies on drilling predation have cast doubt on its validity, at least when drill holes are involved (e.g., Roy et al. 1994; Klompmaker 2009; Smith et al. 2019; Sarkar et al. 2020).

Unlike the extent of time averaging, which can be estimated using temporal information (e.g., carbon isotopes) extracted from specimens and sediments, preservational bias describes the preclusion or removal of information (i.e., individuals) from a sample. It is an estimate of what has been lost and, because it is derived from assumptions and generalized patterns based on characteristics of preserved specimens, cannot be known with absolute certainty. Furthermore, each prey type in a sample may be affected differently by preservational processes based on size, habitat, and other factors. For example, Kosnik et al. (2009) examined the age distributions of four taxa in a death assemblage and, within the same core, found shell half-lives (i.e., the time it takes for half of the shells of a species to be removed from the death assemblage) of 574 (Abranda), 630 (Natica), 925 (Ethalia), and 1229 (Turbo) years. In a core taken nearby, the half-life was 171 years for Abranda and 552 years for Turbo. This variability in cores taken from the same area suggests a need to account for preservational bias, for which Kosnik et al. (2009) suggested a composite shell durability score from shell density, thickness, and shape, to predict shell half-life. The uncertainty introduced by time averaging likely compounds the uncertainty from preservational bias, because the processes contributing to preservational bias are also variable in space and time.

Preservational bias is onerous to estimate and likely contributes to zero inflation and overdispersion in paleoecological count data. The general assumptions, used in many studies, that individuals accumulate at a constant rate and accumulation exceeds removal (Table 2) may not hold (Olszewski 2012; Holland 2016; Tomašových et al. 2016). When estimates on preservational processes are available, they can be readily incorporated into the framework developed here (eq. 7). This hierarchical mixture model approach is well suited to incorporate the uncertainty inherent to paleoecological data by identifying and beginning to account for zeros introduced by ecology and preservation.

\section{Conclusions and Future Work}

Overdispersion and zero inflation are common in paleoecological count data and, just as is the case with ecological count data (MacKenzie et al. 2002; Martin et al. 2005; Blasco-Moreno et al. 2019), can lead to misinterpretation of data and erroneous conclusions. We illustrate this potentiality using data on drilling predation (Martinelli et al. 2015) and time averaging (Kosnik et al. 2009) from molluscan death assemblages as a hypothetical case study. The hierarchical Bayesian framework we present here to analyze these data builds on the foundation of overdispersion and zero-inflation analyses in the ecological literature by allowing for two sources of related count data (i.e., counts of species abundance and predation traces) to be modeled together, rather than from a single source (i.e., counts of species abundance or predation traces) of count data. To the best of our knowledge, this study is the first attempt to incorporate the effects of overdispersion and zero inflation into inferences about predation drawn from paleoecological count data-related methods have been used in occupancy (e.g., Liow 2013) and fossilized birth-death (Barido-Sottani et al. 2019; Warnock et al. 2020) models in paleontology. Though this represents a step forward, it is only a single step among many to be taken to produce more confident ecological interpretations of species' interactions in the paleontological record. Indeed, because of the longer timescales involved, these issues may be more pervasive in fossil assemblages than in the relatively young death assemblages considered here. 
Further work is needed to better account for and understand the complex relationships between overdispersion and zero inflation, and preservational bias and time averaging. Most pressingly, there is a need for paleoecological studies that incorporate estimates of time averaging and preservational bias in the process of drawing inferences, rather than ignoring or dismissing them in advance or checking for their influence after the fact. Without doing so, the long-standing assumptions applied in paleoecology (Table 2), and used here, will continue to limit the inferences drawn from paleoecological data. Collecting data on each of these elements in a single study represents a considerable challenge and deviation from standard practice in the discipline, where these elements are often considered in isolation. With a better accounting of these effects, and thereby uncertainty in paleoecological data, the barriers to integration of data across timescales can more readily be overcome. That is, ecological data and paleoecological data will be more readily comparable, enabling more seamless hypothesis testing across timescales.

It is also important to note that the framework developed here is not one-size-fits-all but can be modified to accommodate different hypotheses. Whereas it would be appropriate to apply the entire framework to evaluate zero inflation in a study examining predator preference, when only ecologically plausible interactions have bearing on the hypothesis (e.g., prey type 1 is preferred over prey type $2)$, it may not be appropriate in a study evaluating mortality risk. Because mortality risk is considered from the perspective of the prey population, a zero for drilling predation should not be excluded, for example, when testing a hypothesis on the relative importance of multiple selective agents (e.g., durophagous predation, drilling predation, abiotic stress). Still, even in the latter case, accounting for uncertainty from time averaging and zero inflation from preservational bias remains relevant, and the models can be adjusted accordingly. As has been stated elsewhere (e.g., Blasco-Moreno et al. 2019), the treatment of zeros in a dataset should be determined by the hypothesis being tested.

Though our case study focused on the effects of overdispersion and zero inflation in paleoecological studies of drilling predation, the modeling framework we developed can be applied to a variety of ecological and paleoecological contexts. Indeed, paleoecological studies examining other predation traces (e.g., repair scars from failed attacks by crabs on clams and snails) or morphological features (e.g., spines, ribs) would likely also benefit from analysis with a hierarchical Bayesian framework that accounts for overdispersion and zero inflation in multiple types of count data. The same is true of ecological studies that make use of more than one type of count data. For example, these methods could be applied to studies of disease prevalence in communities with respect to abundance in the community, the frequency of herbivory on different species in a community with respect to abundance, or any similar set of data with multiple count variables. Ecological and paleoecological inference stand to benefit by more completely addressing the potential for misinterpretation of data caused by overdispersion and zero inflation.

\section{Acknowledgments}

We thank the editors, J. Crampton and J. Huntley, and the reviewers, J. Bauer and P. Novack-Gottshall, for their comments on an earlier version of this article, which improved its quality.

\section{Data Availability Statement}

Data and parameter estimates used in the hypothetical case study (Supplement 1) and code and data files required to reproduce results (Supplement 2) can be found at https://doi.org/10.5281/zenodo.4814590.

\section{Literature Cited}

Adler, P. B., E. P. White, W. K. Lauenroth, D. M. Kaufman, A. Rassweiler, and J. A. Rusak. 2005. Evidence for a general species-time-area relationship. Ecology 86:2032-2039.

Barido-Sottani, J., G. Aguirre-Fernández, M. J. Hopkins, T. Stadler, and R. Warnock. 2019. Ignoring stratigraphic age uncertainty leads to erroneous estimates of species divergence times under the fossilized birth-death process. Proceedings of the Royal Society of London B 286:20190685.

Behrensmeyer, A. K., F. T. Fürsich, R. A. Gastaldo, S. M. Kidwell, M. A. Kosnik, M. Kowalewski, R. E. Plotnick, R. R. Rogers, and J. Alroy. 2005. Are the most durable shelly taxa also the most common in the marine fossil record? Paleobiology 31:607-623. 
Blasco-Moreno, A., M. Pérez-Casany, P. Puig, M. Morante, and E. Castells. 2019. What does a zero mean? Understanding false, random and structural zeros in ecology. Methods in Ecology and Evolution 10:949-959.

Bliss, C. I., and R. A. Fisher. 1953. Fitting the negative binomial distribution to biological data. Biometrics 9:176-200.

Bürkli, A., and A. B. Wilson. 2017. Explaining high-diversity death assemblages: undersampling of the living community, out-of-habitat transport, time-averaging of rare taxa, and local extinction. Palaeogeography, Palaeoclimatology, Palaeoecology 466:174-183.

Cameron, A. C., and P. K. Trivedi. 2013. Regression analysis of count data, Vol. 53. Cambridge University Press, New York.

Chattopadhyay, D., and S. Dutta. 2013. Prey selection by drilling predators: a case study from Miocene of Kutch, India. Palaeogeography, Palaeoclimatology, Palaeoecology 374:187-196.

Chiba, T., and S. Sato. 2014. Invasion of a naticid predator and associated changes in death assemblages of bivalve prey in northern Japan: implications for palaeoecological studies. Lethaia 47:4-14.

Davis, F. M. 1923. Quantitative studies on the fauna of the sea bottom. No. 1, Preliminary investigation of the Dogger Bank. Ministry of Agriculture and Fisheries, Fishery Investigations, London.

Dénes, F. V., L. F. Silveira, and S. R. Beissinger. 2015. Estimating abundance of unmarked animal populations: accounting for imperfect detection and other sources of zero inflation. Methods in Ecology and Evolution 6:543-556.

Dietl, G., and R. Alexander. 1995. Borehole site and prey size stereotypy in naticid predation on Euspira (Lunatia) heros (Say, 1822) and Neverita (Polinices) duplicata (Say, 1822) from the southern New Jersey coast. Journal of Shellfish Research 14:307-314.

Dietl, G. P., and R. R. Alexander. 2000. Post-Miocene shift in stereotypic naticid predation on confamilial prey from the mid-Atlantic shelf: coevolution with dangerous prey. Palaios 15:414-429.

Dyer, A. D., E. R. Ellis, D. J. Molinaro, and L. R. Leighton. 2018. Experimental fragmentation of gastropod shells by sediment compaction: implications for interpreting drilling predation intensities in the fossil record. Palaeogeography, Palaeoclimatology, Palaeoecology 511:332-340.

Flessa, K. W., A. H. Cutler, and K. H. Meldahl. 1993. Time and taphonomy: quantitative estimates of time-averaging and stratigraphic disorder in a shallow marine habitat. Paleobiology 19:266-286.

Gelman, A., and J. Hill. 2006. Data analysis using regression and multilevel/hierarchical models. Cambridge University Press, New York.

Goodwin, D. H., K. W. Flessa, M. A. Téllez-Duarte, D. L. Dettman, B. R. Schöne, and G. A. Avila-Serrano. 2004. Detecting timeaveraging and spatial mixing using oxygen isotope variation: a case study. Palaeogeography, Palaeoclimatology, Palaeoecology 205:1-21.

Hansen, T. A., and P. H. Kelley. 1995. Spatial variation of naticid gastropod predation in the Eocene of North America. Palaios 10:268-278.

Harper, E. M. 2016. Uncovering the holes and cracks: from anecdote to testable hypotheses in predation studies. Palaeontology 59:597-609.

Heilbron, D. C. 1994. Zero-altered and other regression models for count data with added zeros. Biometrical Journal 36:531-547.

Holland, S. M. 2016. The non-uniformity of fossil preservation. Philosophical Transactions of the Royal Society of London B 371:20150130.

Hopkins, M. J., D. W. Bapst, C. Simpson, and R. C. M. Warnock. 2018. The inseparability of sampling and time and its influence on attempts to unify the molecular and fossil records. Paleobiology 44:561-574.

Hougaard, P., M-L T. Lee, and G. A. Whitmore. 1997. Analysis of overdispersed count data by mixtures of Poisson variables and Poisson processes. Biometrics 53:1225-1238.
Johnson, E. H., B. M. Anderson, and W. D. Allmon. 2017. What can we learn from all those pieces? Obtaining data on drilling predation from fragmented high-spired gastropod shells. Palaios 32:271-277.

Johnson, R. G. 1965. Pelecypod death assemblages in Tomales Bay, California. Journal of Paleontology 39:80-85.

Keane, R. M., and M. J. Crawley. 2002. Exotic plant invasions and the enemy release hypothesis. Trends in Ecology and Evolution 17:164-170.

Kelley, P. H. 1989. Evolutionary trends within bivalve prey of Chesapeake Group naticid gastropods. Historical Biology 2:139-156.

Kelley, P. H. 1991. The effect of predation intensity on rate of evolution of five Miocene bivalves. Historical Biology 5:65-78.

Kelley, P. H. 2008. Role of bioerosion in taphonomy: effect of predatory drillholes on preservation of mollusc shells. Pp. 451-470 in M. Wisshak and L. Tapanila, eds. Current developments in bioerosion. Springer-Verlag, Berlin.

Kelley, P. H., and T. A. Hansen. 2003. The fossil record of drilling predation on bivalves and gastropods. Pp. 113-139 in P. H. Kelley, M. Kowalewski, and T. A. Hansen, eds. Predatorprey interactions in the fossil record. Springer, Boston.

Kéry, M. 2010. Introduction to WinBUGS for Ecologists: Bayesian approach to regression, ANOVA, mixed models and related analyses. Academic Press, Boston.

Kidwell, S. M. 2001. Preservation of species abundance in marine death assemblages. Science 294:1091-1094.

Kidwell, S. M. 2002. Time-averaged molluscan death assemblages: palimpsests of richness, snapshots of abundance. Geology 30:803-806

Kidwell, S. M. 2007. Discordance between living and death assemblages as evidence for anthropogenic ecological change. Proceedings of the National Academy of Sciences USA 104:17701-17706. Kidwell, S. M. 2009. Evaluating human modification of shallow marine ecosystems: mismatch in composition of molluscan living and time-averaged death assemblages. Paleontological Society Papers 15:113-139.

Kidwell, S. M. 2013. Time-averaging and fidelity of modern death assemblages: building a taphonomic foundation for conservation palaeobiology. Palaeontology 56:487-522.

Kidwell, S. M., and D. W. J. Bosence. 1991. Taphonomy and timeaveraging of marine shelly faunas. Pp. 115-209 in Taphonomy: releasing the data locked in the fossil record. Topics in Geobiology 9. Plenum Press, New York.

Kitchell, J. A., C. H. Boggs, J. F. Kitchell, and J. A. Rice. 1981. Prey selection by naticid gastropods: experimental tests and application to the fossil record. Paleobiology 7:533-552.

Klompmaker, A. A. 2009. Taphonomic bias on drill-hole predation intensities and paleoecology of Pliocene mollusks from Langenboom (Mill), the Netherlands. Palaios 24:772-779.

Klompmaker, A. A., M. Kowalewski, J. W. Huntley, and S. Finnegan. 2017. Increase in predator-prey size ratios throughout the Phanerozoic history of marine ecosystems. Science 356:1178-1180.

Klompmaker, A. A., P. H. Kelley, D. Chattopadhyay, J. C. Clements, J. W. Huntley, and M. Kowalewski. 2019. Predation in the marine fossil record: studies, data, recognition, environmental factors, and behavior. Earth-Science Reviews 194:472-520.

Korner-Nievergelt, F., T. Roth, S. Von Felten, J. Guélat, B. Almasi, and P. Korner-Nievergelt. 2015. Bayesian data analysis in ecology using linear models with R, BUGS, and Stan. Academic Press, London.

Kosnik, M. A., Q. Hua, D. S. Kaufman, and R. A. Wüst. 2009. Taphonomic bias and time-averaging in tropical molluscan death assemblages: differential shell half-lives in Great Barrier Reef sediment. Paleobiology 35:565-586.

Kowalewski, M., K. W. Flessa, and J. A. Aggen. 1994. Taphofacies analysis of recent shelly cheniers (beach ridges), northeastern Baja California, Mexico. Facies 31:209-242. 
Kowalewski, M., G. A. Goodfriend, and K. W. Flessa. 1998. High-resolution estimates of temporal mixing within shell beds: the evils and virtues of time-averaging. Paleobiology 24:287-304.

Kowalewski, M., S. Casebolt, Q. Hua, K. E. Whitacre, D. S. Kaufman, and M. A. Kosnik. 2018. One fossil record, multiple time resolutions: disparate time-averaging of echinoids and mollusks on a Holocene carbonate platform. Geology 46:51-54.

Lambert, D. 1992. Zero-inflated Poisson regression, with an application to defects in manufacturing. Technometrics 34:1-14.

Leighton, L. R. 2003. Morphological response of prey to drilling predation in the Middle Devonian. Palaeogeography, Palaeoclimatology, Palaeoecology 201:221-234.

Liow, L. H. 2013. Simultaneous estimation of occupancy and detection probabilities: an illustration using Cincinnatian brachiopods. Paleobiology 39:193-213.

MacKenzie, D. I., J. D. Nichols, G. B. Lachman, S. Droege, J. A. Royle, and C. A. Langtimm. 2002. Estimating site occupancy rates when detection probabilities are less than one. Ecology 83:2248-2255.

Mallick, S., S. Bardhan, S. S. Das, S. Paul, and P. Goswami. 2014. Naticid drilling predation on gastropod assemblages across the $\mathrm{K}-\mathrm{T}$ boundary in Rajahmundry, India: new evidence for escalation hypothesis. Palaeogeography, Palaeoclimatology, Palaeoecology 411:216-228.

Martin, T. G., B. A. Wintle, J. R. Rhodes, P. M. Kuhnert, S. A. Field, S. J. Low-Choy, A. J. Tyre, and H. P. Possingham. 2005. Zero tolerance ecology: improving ecological inference by modeling the source of zero observations. Ecology Letters 8:1235-1246.

Martinelli, J. C., M. A. Kosnik, and J. S. Madin. 2015. Encounter frequency does not predict predation frequency in tropical deadshell assemblages. Palaios 30:818-826.

Millar, R. B. 2009. Comparison of hierarchical Bayesian models for overdispersed count data using DIC and Bayes' factors. Biometrics 65:962-969.

Olszewski, T. D. 2012. Remembrance of things past: modeling the relationship between species' abundances in living communities and death assemblages. Biology Letters 8:131-134.

Olszewski, T. D., and S. M. Kidwell. 2007. The preservational fidelity of evenness in molluscan death assemblages. Paleobiology 33:1-23.

Ritter, M. D. N., F. Erthal, M. A. Kosnik, J. C. Coimbra, and D. S. Kaufman. 2017. Spatial variation in the temporal resolution of subtropical shallow-water molluscan death assemblages. Palaios 32:572-583.

Roy, K., D. J. Miller, and M. LaBarbera. 1994. Taphonomic bias in analyses of drilling predation: effects of gastropod drill holes on bivalve shell strength. Palaios 9:413-421.

Sarkar, D., G. Deole, S. Paul, S. Mondal, and R. Saha. 2020. Preburial taphonomic imprints on drilling intensity: a case study from the recent molluscs of Chandipur, India. Historical Biology:1-7.

Sileshi, G. 2008. The excess-zero problem in soil animal count data and choice of appropriate models for statistical inference. Pedobiologia 52:1-17.

Sime, J. A., and P. H. Kelley. 2016. Common mollusk genera indicate interactions with their predators were ecologically stable across the Plio-Pleistocene extinction. Palaeogeography, Palaeoclimatology, Palaeoecology 463:216-229.

Smith, J. A., and G. P. Dietl. 2016. The value of geohistorical data in identifying a recent human-induced range expansion of a predatory gastropod in the Colorado River delta, Mexico. Journal of Biogeography 43:791-800.

Smith, J. A., J. C. Handley, and G. P. Dietl. 2018a. On drilling frequency and Manly's alpha: towards a null model for predator preference in paleoecology. Palaios 33:61-68.

Smith, J. A., J. C. Handley, and G. P. Dietl. 2018b. Effects of dams on downstream molluscan predator-prey interactions in the Colorado River estuary. Proceedings of the Royal Society of London B 285:20180724.

Smith, J. A., G. P. Dietl, and J. C. Handley. 2019. Durophagy bias: the effect of shell destruction by crushing predators on drilling frequency. Palaeogeography, Palaeoclimatology, Palaeoecology 514:690-694.

Sólymos, P., S. Lele, and E. Bayne. 2012. Conditional likelihood approach for analyzing single visit abundance survey data in the presence of zero inflation and detection error. Environmetrics 23:197-205.

Stafford, E. S., C. L. Tyler, and L. R. Leighton. 2015. Gastropod shell repair tracks predator abundance. Marine Ecology 36:1176-1184.

Stan Development Team. 2018. RStan: the R interface to Stan, R Package Version 2.18.2. http://mc-stan.org.

Stan Development Team. 2019. Stan modeling language users guide and reference manual, Version 2.26. https://mc-stan.org/docs / 2_26/stan-users-guide/index.html.

Tomašovỳch, A., and S. M. Kidwell. 2009. Fidelity of variation in species composition and diversity partitioning by death assemblages: time-averaging transfers diversity from beta to alpha levels. Paleobiology 35:94-118.

Tomašovỳch, A., and S. M. Kidwell. 2010. Predicting the effects of increasing temporal scale on species composition, diversity, and rank-abundance distributions. Paleobiology 36:672-695.

Tomašovỳch, A., S. M. Kidwell, and R. F. Barber. 2016. Inferring skeletal production from time-averaged assemblages: skeletal loss pulls the timing of production pulses towards the modern period. Paleobiology 42:54-76.

van de Schoot, R., S. Depaoli, R. King, B. Kramer, K. Märtens, M. G. Tadesse, M. Vannucci, A. Gelman, D. Veen, and J. Willemsen. 2021. Bayesian statistics and modeling. Nature Reviews Methods Primers 1:1-26.

Vehtari, A., A. Gelman, and J. Gabry. 2017. Practical Bayesian model evaluation using leave-one-out cross-validation and WAIC. Statistics and Computing 27:1413-1432.

Vermeij, G. J. 1987. Evolution and escalation: an ecological history of life. Princeton University Press, Princeton, N.J.

Walker, S. E. 1989. Hermit crabs as taphonomic agents. Palaios 4:439-452.

Warme, J. E. 1969. Live and dead molluscs in a coastal lagoon. Journal of Paleontology 43:141-150.

Warnock, R. C. M., T. A. Heath, and T. Stadler. 2020. Assessing the impact of incomplete species sampling on estimates of speciation and extinction rates. Paleobiology 46:137-157.

Warton, D. I. 2005. Many zeros does not mean zero inflation: comparing the goodness-of-fit of parametric models to multivariate abundance data. Environmetrics 16:275-289.

Welsh, A. H., R. B. Cunningham, C. F. Donnelly, and D. B. Lindenmayer. 1996. Modeling the abundance of rare species: statistical models for counts with extra zeros. Ecological Modeling 88:297-308.

Wenger, S. J., and M. C. Freeman. 2008. Estimating species occurrence, abundance, and detection probability using zero-inflated distributions. Ecology 89:2953-2959. 